\title{
Coherent phonon lasing in a thermal quantum nanomachine
}

\author{
P. Karwat,,${ }^{1,2, *}$ D. E. Reiter, ${ }^{2,3}$ T. Kuhn, ${ }^{3}$ and O. Hess ${ }^{2, \dagger}$ \\ ${ }^{1}$ Department of Theoretical Physics, Wroctaw University of Science and Technology, Wybrzeże Wyspianskiego 27, 50-370 Wroctaw, Poland \\ ${ }^{2}$ The Blackett Laboratory, Department of Physics, Imperial College London, South Kensington Campus, SW7 2AZ London, United Kingdom \\ ${ }^{3}$ Institut für Festkörpertheorie, Westfälische Wilhelms-Universität Münster, Wilhelm-Klemm-Strasse 10, 48149 Münster, Germany
}

(Received 6 September 2018; published 29 November 2018)

\begin{abstract}
The notion of nanomachines has recently emerged to engage and use collective action of ensembles of nanoscale components or systems. Here we present a heat-gradient driven nanomachine concept which through appropriate coupling between quantum nanosystems is capable of realizing and maintaining an inversion. Based on a Lindblad form of the quantum master equation with a semiclassical coupling to the lattice displacement phonon field we show that this positive inversion can be harnessed to generate coherent optomechanical oscillations and phonon lasing.
\end{abstract}

DOI: 10.1103/PhysRevA.98.053855

\section{INTRODUCTION}

In nature, an inversion of the occupation probabilities of quantum states is clearly quite exotic since the standard thermal situation leads to the very opposite-i.e., to a Boltzmann distribution of the energy levels in which the lower levels are populated exponentially larger than higher ones. Typically such an inversion is achieved by pumping the system, e.g., electrically or optically. Once achieved, an inversion will allow an initially very small field to be amplified by several orders of magnitude paving the route to lasing. This prompts the question of whether it is possible to use a simple temperature difference not only to drive a system such as a steam engine, but also to promote coherent amplification and lasing.

In this paper, we will address this matter by considering a nanomachine working with and emitting phonons (rather than, e.g., photons), a concept which has recently been the focus of a number of studies [1-6]. We will, in particular, explore if there is a possibility of an amplification of phonon waves, which in analogy to optical lasing, could lead to the concept of a phonon-laser or saser [7,8] used, e.g., in innovative highly precise nondestructive measurements [9]. Not surprisingly, several proposals for phonon lasing have been suggested, e.g., schemes based on optomechanical systems $[1,3,6,10]$ or on semiconductor quantum-well structures [11]. Here, we will aim to establish a route to phonon lasing on the nanoscale, in a nanomachine driven by a heat gradient.

\section{NANOMACHINE SYSTEM}

Our nanomachine is composed of three coupled quantum systems (QSs), which are subject to a heat gradient as displayed in Fig. 1(a). The active medium is the middle threelevel system (QS M). As we shall discuss in further detail in the following, this central quantum system interacts with twolevel subsystems (QS L/R) at each side, which act as energy filters. Such filtering is necessary, because a direct coupling

\footnotetext{
*Corresponding author: Pawel.Karwat@pwr.edu.pl

${ }^{\dagger}$ Corresponding author: O.Hess@imperial.ac.uk
}

to the two heat baths (each with a different temperature) would lead to a thermal occupation of the system and not to an inversion. In our nanomachine each filter is coupled to a heat bath, where the left bath has a significantly higher temperature $\left(T_{\mathrm{H}}\right)$ than the right one $\left(T_{\mathrm{C}}\right)$. As a consequence of the temperature difference, a flow of excitation takes place. We will show that for characteristic parameters the exclusive thermalization of the resonant transitions may lead to a crucial inversion in the upper two levels of the central system. The inversion can then result in the emission of coherent phonons at the central quantum system.

The Hilbert space of the nanomachine is spanned by the product of the basis states in the QSs as depicted in Fig. 1(a). The relative energies of the states in the two-level systems are parametrized by $\delta_{\mathrm{L} / \mathrm{R}}$ and in the central three-level system by $\delta_{\mathrm{M}_{+} / \mathrm{M}_{-}}$. The system Hamiltonian reads

$$
\hat{H}_{\text {sys }}=\hat{h}^{(\mathrm{L})}+\hat{h}^{(\mathrm{M})}+\hat{h}^{(\mathrm{R})},
$$

where $\hat{h}^{(\mathrm{L}) /(\mathrm{M}) /(\mathrm{R})}$ are the Hamiltonians of the QSs with

$$
\hat{h}^{(\mathrm{L})}=\sum_{n} \epsilon_{n}^{(\mathrm{L})} P_{n n}^{(\mathrm{L})} \otimes \hat{1}^{(\mathrm{M})} \otimes \hat{1}^{(\mathrm{R})} .
$$

Here, $\epsilon_{n}^{(\mathrm{L})}$ are the energies, $\hat{P}_{n m}^{(\mathrm{L})}=|n\rangle^{(\mathrm{L})(\mathrm{L})}\langle m|$ is the projection operator, and $\hat{1}^{(\mathrm{M}) /(\mathrm{R})}$ denote unity operators in the space of the respective subsystem. The Hamiltonians $\hat{h}^{(\mathrm{M})}$ and $\hat{h}^{(\mathrm{R})}$ are constructed in the same way.

The QSs are coupled in a way that excitations can be exchanged between adjacent sites as denoted in Fig. 1(a), i.e., the middle system interacts with both sides, while the direct interactions between the left and right system is suppressed. The corresponding Hamiltonian reads

$$
\hat{H}_{\text {int }}=\lambda_{\mathrm{ML}}\left(\hat{h}^{(\mathrm{LM})} \otimes \hat{1}^{(\mathrm{R})}\right)+\lambda_{\mathrm{MR}}\left(\hat{1}^{(\mathrm{L})} \otimes \hat{h}^{(\mathrm{MR})}\right),
$$

where $\lambda_{\mathrm{ML} / \mathrm{MR}}$ is the coupling parameter. The coupling

$$
\hat{h}^{(\mathrm{LM})}=\hat{P}_{21}^{(\mathrm{L})} \otimes\left(\hat{P}_{12}^{(\mathrm{M})}+\hat{P}_{13}^{(\mathrm{M})}+\hat{P}_{23}^{(\mathrm{M})}\right)+\text { H.c. }
$$

is given by the respective projection operators and analogous for $\hat{h}^{(\mathrm{MR})}$. The coupling is taken to be weak such that the energy contribution of the interaction is small compared to the energy contained in the system. 


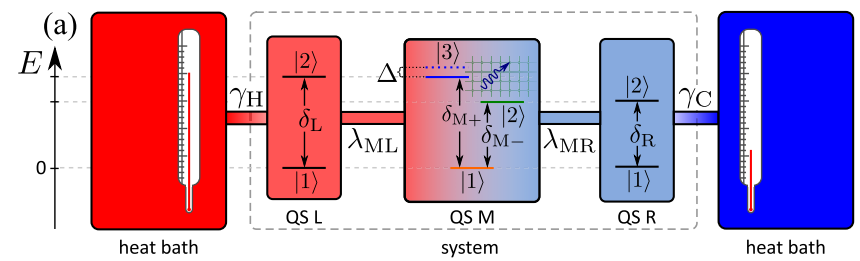

(b)

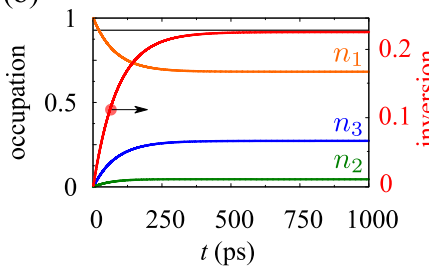

(c)

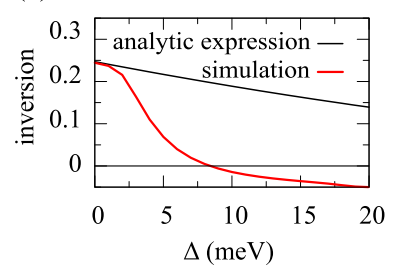

FIG. 1. (a) Schematic setup of the thermally driven nanomachine. (b) Occupations $n_{i}$ of the middle QS as function of time for temperatures $T_{\mathrm{H}}=400 \mathrm{~K}$ and $T_{\mathrm{C}}=100 \mathrm{~K}$ as well as the inversion $I=n_{3}-n_{2}$. (c) Inversion as function of energy mismatch $\Delta$. The dashed lines marks an analytic expression of the (ideal) inversion according to Eq. (9).

Each of the two edge QSs is coupled locally to a heat bath of different temperature. To describe the coupling, we make use of a quantum master equation within a Lindblad form, which accounts for the nonequilibrium situation in our system [12]. For this, we set up the equation of motion for the density matrix $\hat{\rho}$ via

$$
\frac{d \hat{\rho}}{d t}=-\frac{i}{\hbar}\left[\hat{H}_{\mathrm{sys}}+\hat{H}_{\mathrm{int}}, \hat{\rho}\right]+\hat{D}_{\mathrm{H}}(\hat{\rho})+\hat{D}_{\mathrm{C}}(\hat{\rho}) .
$$

$\hat{D}_{\mathrm{H}}$ and $\hat{D}_{\mathrm{C}}$ are the dissipators to the hot (left) and cold (right) heat bath, respectively,

$$
\hat{D}_{\mathrm{H}}(\hat{\rho})=\sum_{k=1}^{2} \Gamma_{k}\left(T_{\mathrm{H}}\right)\left(\hat{L}_{k}^{(\mathrm{L})} \hat{\rho} \hat{L}_{k}^{(\mathrm{L}) \dagger}-\frac{1}{2}\left[\hat{L}_{k}^{(\mathrm{L}) \dagger} \hat{L}_{k}^{(\mathrm{L})}, \hat{\rho}\right]_{+}\right),
$$

with the Lindblad operators

$$
\hat{L}_{1}^{(\mathrm{L})}=\hat{P}_{21}^{(\mathrm{L})} \otimes \hat{1}^{(\mathrm{M}, \mathrm{R})}, \quad \hat{L}_{2}^{(\mathrm{L})}=\hat{P}_{12}^{(\mathrm{L})} \otimes \hat{1}^{(\mathrm{M}, \mathrm{R})} .
$$

The effectiveness of the heat coupling is given by the rates $\Gamma_{k}$ chosen by a phenomenological ansatz for the spectral density of an environment of Ohmic kind [12] with

$$
\Gamma_{k}\left(T_{\mathrm{H}}\right)=\frac{\gamma}{1+\exp \left\{(-1)^{k-1} \delta_{\mathrm{L}} / k_{\mathrm{B}} T_{\mathrm{H}}\right\}},
$$

containing the distribution function. The dissipator describing the coupling to the cold (right) heat bath $\hat{D}_{\mathrm{C}}(\hat{\rho})$ is analog.

Our goal is to achieve an inversion between states $|3\rangle^{(\mathrm{M})}$ and $|2\rangle^{(\mathrm{M})}$, which later will be coupled to a phonon mode. The energy of typical acoustic phonons lies in the order of a few meV. Setting the energy of the lower state $|1\rangle^{(\mathrm{M})}$ to zero, we accordingly chose $\delta_{\mathrm{M}_{+}}=30 \mathrm{meV}$ and $\delta_{\mathrm{M}_{-}}=25$ meV, thus $\delta_{\mathrm{M}_{+}}-\delta_{\mathrm{M}_{-}}=5 \mathrm{meV}$. The energies of the edge states are set to $\delta_{\mathrm{L}}=\delta_{\mathrm{M}_{+}}=30 \mathrm{meV}$ and $\delta_{\mathrm{R}}=\delta_{\mathrm{M}_{-}}=25$ meV. If not stated otherwise, the parameters are set to $\lambda=$ $\lambda_{\mathrm{ML}}=\lambda_{\mathrm{MR}}=0.03 \mathrm{meV}, \gamma=\gamma_{\mathrm{H}}=\gamma_{\mathrm{C}}=3 \mathrm{ps}^{-1}$. As initial condition we assume that the whole system is in its ground state, $|1\rangle^{(\mathrm{L})} \otimes|1\rangle^{(\mathrm{M})} \otimes|1\rangle^{(\mathrm{R})}$.



FIG. 2. (a) Inversion as function of temperature difference $\Delta T$ for a constant temperature of the colder bath $T_{\mathrm{C}}=100,200$, and $300 \mathrm{~K}$. (b) Color map of the inversion as a function of temperature difference and the mean temperature of the system $T_{\text {sys }}=\left(T_{\mathrm{H}}+\right.$ $\left.T_{\mathrm{C}}\right) / 2$. The symbols (white circle, pentagon, and triangle) correspond to the curves from the upper panel. The yellow line depicts zero inversion.

\section{INVERSION IN THE NANOMACHINE}

By solving the equation of motion we calculate the occupations $n_{i}=\left\langle P_{i i}^{(\mathrm{M})}\right\rangle$ of the three states in QS M. The time evolution of the occupations for a hot bath with temperature $T_{\mathrm{H}}=400 \mathrm{~K}$ and a cold bath of $T_{\mathrm{C}}=100 \mathrm{~K}$ is shown in Fig. 1(b). When the heating gradient is switched on at $t=0$, the occupation $n_{1}$ decreases in favor of $n_{2}$ and $n_{3}$. After a few hundreds of picoseconds a stationary state is reached. Due to the heat gradient and the energy filtering an inversion in the central system is achieved (red solid line). It is interesting to compare the achieved inversion to the ideal case in which the occupations $n_{i}$ will follow the Boltzmann distribution,

$$
\frac{n_{3}}{n_{1}}=\exp \left\{-\frac{\delta_{\mathrm{M}+}}{k_{\mathrm{B}} T_{\mathrm{H}}}\right\}, \quad \frac{n_{2}}{n_{1}}=\exp \left\{-\frac{\delta_{\mathrm{M}-}}{k_{\mathrm{B}} T_{\mathrm{C}}}\right\} .
$$

The inversion in the ideal case is then

$$
I=n_{3}-n_{2}=\frac{A-B}{1+A+B},
$$

where $A=\exp \left\{-\frac{\delta_{\mathrm{M}+}}{k_{\mathrm{B}} T_{\mathrm{H}}}\right\}$ and $B=\exp \left\{-\frac{\delta_{\mathrm{M}-}}{k_{\mathrm{B}} T_{\mathrm{C}}}\right\}$. Inserting the $T_{\mathrm{H}}=400 \mathrm{~K}$ and $T_{\mathrm{C}}=100 \mathrm{~K}$ into the equation we obtain an inversion of $I^{\text {ideal }}=0.247$, which is slightly above the numerically calculated value of $I^{\text {num }}=0.244$. Hence, we can conclude that through the filters the heat gradient applied at the edge systems leads to an inversion in the middle system.

The creation of an inversion depends sensitively on the energy filters as seen when introducing an energy mismatch $\Delta$ between the filter systems and the middle system in Fig. 1(c). 

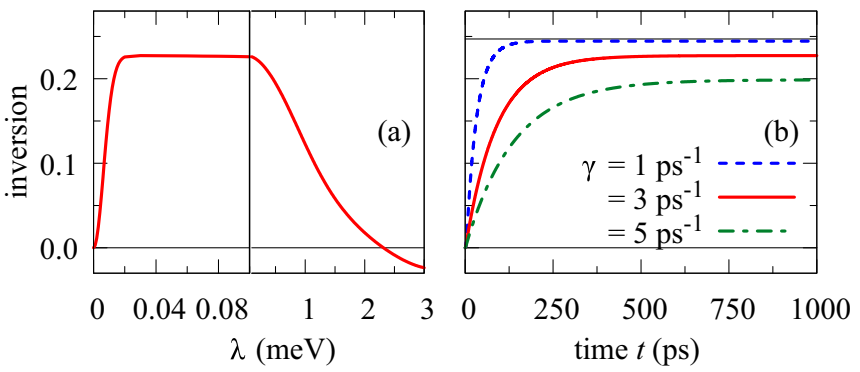

FIG. 3. Stationary values of the inversion as function of (a) QS coupling parameter $\lambda$ and (b) heath bath coupling constant $\gamma$. The bath temperatures are $T_{\mathrm{H}}=400 \mathrm{~K}$ and $T_{\mathrm{C}}=100 \mathrm{~K}$. The thin black line marks the ideal inversion.

Note that the mismatch is given such that the energy of state $|3\rangle^{(M)}$ increases to $\delta_{M+}+\Delta$. With increasing $\Delta$ the inversion decreases dramatically and for $\Delta>8 \mathrm{meV}$ it changes its sign returning to a normal condition.

Establishing that a simple heat gradient is, indeed, upon application of appropriate filters able to create a population inversion, we now consider the range of temperatures for which a population inversion is possible. Figure 2(a) shows for fixed temperature of the cold bath $T_{\mathrm{C}}$ the dependence of the inversion on the temperature difference $\Delta T=T_{\mathrm{H}}-T_{\mathrm{C}}$. As expected, for higher temperature differences $\Delta T$ the inversion increases. However, an inversion is only reached over a certain threshold given by the ratio $T_{\mathrm{H}} / T_{\mathrm{C}}>1.2$. This threshold depends not only on the temperature difference, but also on the absolute values of the baths. Figure 2(b) shows a color representation of the inversion as a function of the temperature difference of the heat baths (for the wider temperature range) and the mean temperature of the system defined as $T_{\text {sys }}=$ $\left(T_{\mathrm{H}}+T_{\mathrm{C}}\right) / 2$. The results in Fig. 2(b) clearly underline the fact that a minimal temperature difference is needed to achieve inversion.

The inversion is also sensitive to other system parameters, like system coupling parameter $\lambda$ or the heat bath coupling parameter $\gamma$. Figure 3(a) displays the stationary inversion as function of $\lambda$ while keeping all other parameters fixed. The temperatures are $T_{\mathrm{H}}=400 \mathrm{~K}$ and $T_{\mathrm{C}}=100 \mathrm{~K}$. For small values of $\lambda$ the inversion increases, reflecting that an increasingly efficient heat coupling enables an increasingly efficient transfer of the excitation to the center system. Subsequently, the inversion saturates and remains constant over a large range of $\lambda$ up until a point when for high values of $\lambda$ the inversion decreases again. At this point, the interaction between the systems is not weak anymore and the new eigenstates become a superposition of the uncoupled states. In Fig. 3(b) we present the impact of the coupling of each two-level system to their environment on the inversion. The parameter $\gamma$ determines how fast the edge systems, and subsequently the middle system, are thermalized. With a higher value of $\gamma$ the thermalization becomes faster and as a consequence the inversion reaches nearly the ideal value of $I^{\text {ideal }}$ marked by the black line [cf. Eq. (9)].
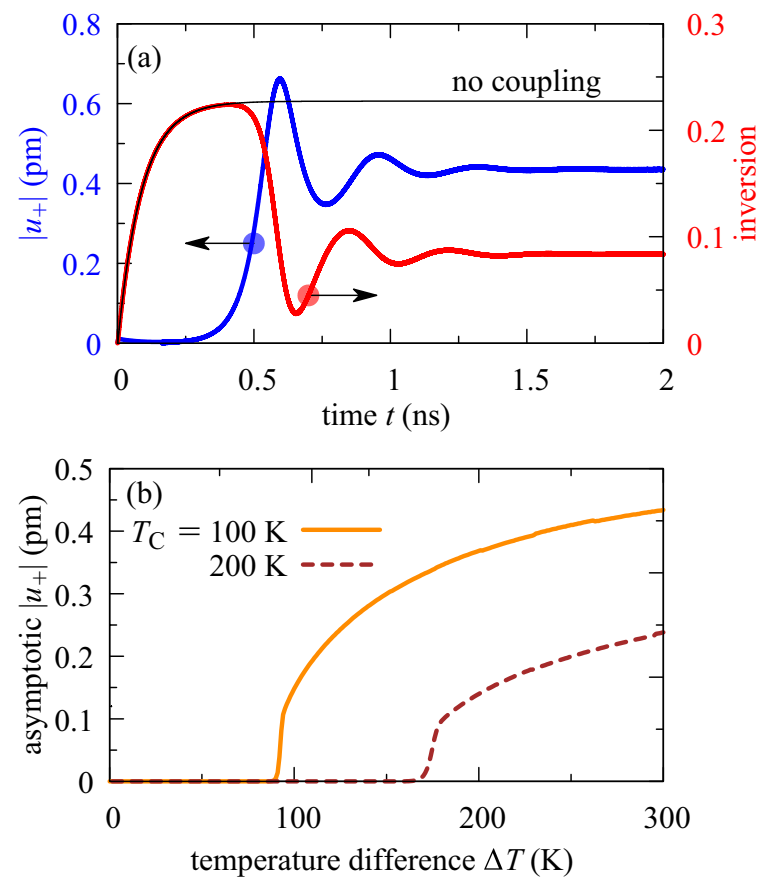

FIG. 4. (a) Phonon-lasing relaxation oscillations of the inversion (red) and the amplitude of the lattice displacement field (blue) for $T_{\mathrm{H}}=400 \mathrm{~K}$ and $T_{\mathrm{C}}=100 \mathrm{~K}$. (b) Stationary amplitude of the lattice displacement field as function of temperature difference for $T_{\mathrm{C}}=$ $100 \mathrm{~K}$ (orange) and $T_{\mathrm{C}}=200 \mathrm{~K}$ (brown).

\section{PHONON LASING}

Having seen that the heat gradient does in our simple nanomachine, indeed, induce an inversion, we are now ready to test if this inversion can actually be utilized for a phonon laser. For simplicity, we assume a single acoustic-phonon mode, as realized in a cavity formed, e.g., in semiconductor superlattices [13-16]. While in the following embracing the characteristic properties of phonons in coupled semiconductor quantum systems, our concept can readily be transferred to optomechanical systems involving phonon lasing in nanomechanical oscillators [17-19]. Denoting $b, b^{\dagger}$ as the bosonic operators of the phonon mode and $\omega$ its frequency, the phonon Hamiltonian is composed of the free phonon system and the carrier-phonon coupling

$$
\hat{H}_{\mathrm{ph}}=\hbar \omega \hat{b}^{\dagger} \hat{b}+\hat{H}_{\mathrm{c}-\mathrm{ph}} .
$$

The energy of the phonon is chosen to be resonant with the energy difference in QS M with $\hbar \omega=5 \mathrm{meV}$. The phonon mode is coupled only to the middle QS M via

$$
\hat{H}_{\mathrm{c}-\mathrm{ph}}=\hat{1}^{(\mathrm{L})} \otimes \hat{h}^{(\mathrm{M})} \otimes \hat{1}^{(\mathrm{R})}
$$

with

$$
\hat{h}^{(\mathrm{M})}=\hbar g\left(\hat{b}^{\dagger} \hat{P}_{23}^{(\mathrm{M})}+\hat{b} \hat{P}_{32}^{(\mathrm{M})}\right) .
$$

Here $g$ is the real coupling constant between the QS and phonon mode. We assume that the system can relax from the state $|3\rangle^{(\mathrm{M})}$ to state $|2\rangle^{(\mathrm{M})}$ by the emission of a phonon, while the reverse transition is possible by absorbing a phonon. 
A measurable quantity for the phonons is the lattice displacement, which is linked to the phonon operators via

$$
\langle\hat{u}\rangle=u_{0}\left(\left\langle\hat{b}^{\dagger}\right\rangle+\langle\hat{b}\rangle\right)=u^{(+)}+u^{(-)},
$$

where we defined $u^{(+)}=u_{0}\langle\hat{b}\rangle$ and $u^{(-)}=u_{0}\left\langle\hat{b}^{\dagger}\right\rangle$ as well as the single phonon amplitude $u_{0}$.

Introducing the phonon coupling, we extend the equations of motion to

$$
\frac{d \hat{\rho}}{d t}=-\frac{i}{\hbar}\left[\hat{H}_{\mathrm{sys}}+\hat{H}_{\mathrm{int}}+\hat{H}_{\mathrm{ph}}, \hat{\rho}\right]+\hat{D}_{\mathrm{H}}(\hat{\rho})+\hat{D}_{\mathrm{C}}(\hat{\rho}) .
$$

For the lattice displacement this leads to the following rate equation:

$$
\frac{d u}{d t}=-\Gamma u-i C\left(\rho_{23}^{(\mathrm{M})}(t)+\rho_{32}^{(\mathrm{M})}(t)\right),
$$

where we introduced the phonon dephasing rate $\Gamma$ and defined the coupling constant $C=u_{0} g$. For our simulations we assume $\Gamma=2 \mathrm{ps}^{-1}, g=2.25 \mathrm{ps}^{-1}, u_{0}=20 \mathrm{pm}$. We further assume that always a very small, but finite displacement is present.

Figure 4(a) shows the evolution of the inversion (red) in comparison to the amplitude of the lattice displacement field (blue). The heat-bath temperatures are set to $T_{\mathrm{H}}=400 \mathrm{~K}$ and $T_{\mathrm{C}}=100 \mathrm{~K}$. We observe phonon lasing relaxation-oscillation dynamics similar to the well-known optical relaxation oscillations of conventional optical semiconductor lasers. For small times the inversion of the system builds up according to the thermalization of the middle QS M. When the inversion is close to its maximum the lattice displacement starts to increase. Then the inversion and the lattice pitched against each other in the relaxation oscillations. After some time a steady state is reached with a lattice displacement of $u_{\infty}=0.43 \mathrm{pm}$. In this quasisteady state a constant flow of coherent phonons occurs-phonon lasing. In Fig. 4(b), we show the amplitude of the phonon flow as a function of the temperature difference $\Delta T$. Keeping the cold bath fixed at $T_{\mathrm{C}}=100 \mathrm{~K}$ (solid line), we increase the hot bath temperature. For a classical laser such an output curve should exhibit a characteristic onset of lasing at a given threshold [20]. Indeed, we find a characteristic onset of phonon lasing at $\Delta T_{\mathrm{on}}=85 \mathrm{~K}$, where for temperature difference below $\Delta T_{\text {on }}$ no phonons are emitted and the phonon amplitude rises significantly for temperature differences above $\Delta T_{\text {on }}$ as expected for lasing. Like the inversion, the threshold depends not only on the temperature difference, but also on the absolute values of the temperature. If we fix the temperature of the cold bath to $T_{\mathrm{C}}=200 \mathrm{~K}$ (dashed line), the threshold increases to $\Delta T_{\mathrm{on}}^{\prime}=150 \mathrm{~K}$.

\section{CONCLUSIONS}

We have presented and studied the properties of a heatgradient driven nanomachine concept which, through appropriate coupling between quantum nanosystems, is capable of realizing and maintaining an inversion. Based on a Lindblad form of the quantum master equation with a semiclassical coupling to lattice displacement phonons we have shown that this positive inversion can be harnessed to generate coherent optomechanical oscillations and phonon lasing. Remarkably, despite the opening of a strong heat conducting channel between the hot and the cold reservoirs due to the phononlasing action, the system shows an amplification of the lattice amplitude and displays laser characteristics such as dynamic relaxation oscillations of the phonon displacement amplitude and the inversion as well as a pronounced threshold behavior.
[1] J. Zhang, B. Peng, S. K. Ozdemir K. Pichler, D. O. Krimer, G. Zhao, F. Nori, Y. Liu, S. Rotter, and L. Yang, A phonon laser operating at an exceptional point, Nat. Photon. 12, 479 (2018).

[2] L. Droenner, N. L. Naumann, J. Kabuss, and A. Carmele, Collective enhancements in many-emitter phonon lasing, Phys. Rev. A 96, 043805 (2017).

[3] K. Vahala, M. Herrmann, S. Knunz, V. Batteiger, G. Saathoff, T. W. Hansch, and Th. Udem, A phonon laser, Nat. Phys. 5, 682 (2009).

[4] J. T. Mendonça, H. Terças, G. Brodin, and M. Marklund, A phonon laser in ultra-cold matter, Europhys. Lett. 91, 33001 (2010).

[5] K. V. Kepesidis, S. D. Bennett, S. Portolan, M. D. Lukin, and P. Rabl, Collective enhancements in many-emitter phonon lasing, Phys. Rev. B 88, 064105 (2013).

[6] I. Mahboob, K. Nishiguchi, A. Fujiwara, and H. Yamaguchi, Phonon Lasing in an Electromechanical Resonator, Phys. Rev. Lett. 110, 127202 (2013).

[7] S. T Zavtrak and I. V. Volkov, Saser (sound amplification by stimulated emission of radiation), Tech. Phys. 42, 406 (1997).

[8] L. G. Tilstra, A. F. M. Arts, and H. W. de Wijn, Optically excited ruby as a saser: experiment and theory, Phys. Rev. B 76, 024302 (2007).
[9] J. B. Khurgin, Viewpoint: Phonon lasers gain a sound foundation, Physics 3, 16 (2010).

[10] K. Sandner and H. Ritsch, Temperature Gradient Driven Lasing and Stimulated Cooling, Phys. Rev. Lett. 109, 193601 (2012).

[11] W. Maryam, A. V. Akimov, R. P. Campion, and A. J. Kent, Dynamics of a vertical cavity quantum cascade phonon laser structure, Nat. Commun. 4, 2184 (2013).

[12] H. P. Breuer and F. Petruccione, The Theory of Open Quantum Systems (Oxford University Press, Oxford, 2002).

[13] M. Trigo, A. Bruchhausen, A. Fainstein, B. Jusserand, and V. Thierry-Mieg, Confinement of Acoustical Vibrations in a Semiconductor Planar Phonon Cavity, Phys. Rev. Lett. 89, 227402 (2002).

[14] N. D. Lanzillotti-Kimura, A. Fainstein, C. A. Balseiro, and B. Jusserand, Phonon engineering with acoustic nanocavities: Theoretical considerations on phonon molecules, band structures, and acoustic bloch oscillations, Phys. Rev. B 75, 024301 (2007).

[15] J. Kabuss, A. Carmele, T. Brandes, and A. Knorr, Optically Driven Quantum Dots as Source of Coherent Cavity Phonons: a Proposal for a Phonon Laser Scheme, Phys. Rev. Lett. 109, 054301 (2012).

[16] M. Esmann, F. R. Lamberti, P. Senellart, I. Favero, O. Krebs, L. Lanco, C. G. Carbonell, A. Lemaître, and N. D. Lanzillotti- 
Kimura, Topological nanophononic states by band inversion, Phys. Rev. B 97, 155422 (2018).

[17] T. J. Kippenberg and K. J. Vahala, Cavity opto-mechanics, Opt. Express 15, 17172 (2007).

[18] M. Aspelmeyer, T. J. Kippenberg, and F. Marquardt, Cavity optomechanics, Rev. Mod. Phys. 86, 1391 (2014).
[19] G. Anetsberger, O. Arcizet, Q. P. Unterreithmeier, R. Rivière, A. Schliesser, E. M. Weig, J. P. Kotthaus, and T. J. Kippenberg, Near-field cavity optomechanics with nanomechanical oscillators, Nat. Phys. 5, 909 (2009).

[20] G. Björk, A. Karlsson, and Y. Yamamoto, Definition of a laser threshold, Phys. Rev. A 50, 1675 (1994). 